The use of targeted and immunotherapies was not common perhaps because the approval of these treatments was recent and not adequately captured in the data. Delaying progression to subsequent LOTs may help reduce the economic burden in this population.

Disclosures This study was funded by Merck Sharp \& Dohme Corp., a subsidiary of Merck \& Co., Inc., Kenilworth, NJ, USA. Chizoba Nwankwo is an employee of Merck Sharp \& Dohme Corp., a subsidiary of Merck \& Co., Inc. Anuj Shah, Ruchit Shah, Shelby Corman, and Nehemiah Kebede are employees of Pharmerit, which received consulting fees related to this study.

\section{PROGNOSTIC FACTORS FOR LOCALIZED UTERINE CARCINOSARCOMA - 18 YEARS OF REAL-WORLD PRACTICE OF A PORTUGUESE CANCER CENTRE}

${ }^{1}$ Catarina Bexiga, ${ }^{2}$ Francisca Gonçalves, ${ }^{2}$ Adriana Ramos, ${ }^{2}$ Ana Clara, ${ }^{2}$ Fátima Vaz. 'Instituto Português de Oncologia de Lisboa, Francisco Gentil; Oncologia; 'Instituto Português de Oncologia de Lisboa, Francisco Gentil

\subsection{6/ijgc-2020-ESG0.65}

Introduction/Background Uterine carcinosarcoma (UCS) is a rare but aggressive malignancy. It represents $5 \%$ all of uterine tumors and is responsible for $30 \%$ of uterine cancer deaths. Known risk factors for UCS are age, pelvic irradiation, and tamoxifen use. Prognostic factors are not clearly defined. This study aims to determine prognostic factors for survival in UCS.

Methodology Observational retrospective study of pts with UCS treated in a Cancer Centre between 2000-2018. Clinical data was retrieved from records. Prognostic variables were tested by multivariate analysis using Cox's proportional hazards regression model, and Kaplan-Meier survival curves were generated.

Results A total of 73 women with early or locally advanced UCS were identified, with median age 68.0 yrs (46-89). Most pts had Performance Status (PS) $0-1 \quad(n=59,80.8 \%)$. Regarding predisposing factors, 8 had used tamoxifen and 5 had underwent pelvic radiotherapy. FIGO stage distribution as follows: 26 (35.5\%) stage I; 13 (17.7\%) stage II; 30 (41.0\%) stage III; and $4(5.8 \%)$ stage IVA.

Initial treatment was surgery for 70 pts. All pts underwent total hysterectomy and bilateral anexectomy, 22 (31.4\%) pts pelvic and lomboaortic lymph node dissection (LND), and 19 (27.1\%) pts isolated pelvic LND. Residual disease was present in 15 pts (20.5\%). Adjuvant treatment was prescribed as follows: isolated radiotherapy (RT) for 22 pts (30.1\%) (of which 13 received additional brachytherapy), chemotherapy followed by RT for 17 pts (23.3\%) and isolated chemotherapy for 11 pts (15.1\%). Isolated adjuvant RT was prescribed mostly before 2010, and afterwards the use of adjuvant chemotherapy became more common.

After a median follow up of 29.7 months (95\% CI [22.1$37.4]), 51$ pts (69.9\%) died. Relapse occurred in 40 pts $(54.8 \%)$, mostly with a pattern of distant failure (33 pts). Local recurrence occurred in 18 pts. Median overall survival (OS) and disease free survival (DFS) were 18.3 (95\% CI 13.3-23.3) and 11.3 (95\% CI 7.5-15) months, respectively.

In multivariate analysis, PS (HR 3.93, 95\% CI [1.1613.27], $\mathrm{p}=0.028$ ), residual disease (HR 12.21, 95\% CI [2.13$70.02], \mathrm{p}=0.005$ ), adjuvant RT (HR 0.27, 95\% CI [0.09-
0.83], $\mathrm{p}=0.022$ ) and adjuvant brachytherapy (HR 0.31, 95\% CI [0.09-0.99], $\mathrm{p}=0.048$ ) were independent prognostic factors for OS. No prognostic factors for DFS were found.

Conclusion In concordance with previous studies, UCS presented a high rate of recurrence and mortality. This study identified PS, residual disease, and adjuvant radiotherapy and brachytherapy as prognostic factors for OS. Despite relapse occurring mostly at distance, adjuvant chemotherapy did not impact survival.

Disclosures The authors have no disclosures.

\section{SENTINEL LYMPH-NODE MAPPING WITH INDOCYANINE GREEN IN ENDOMETRIAL CANCER: DETECTION RATE AND ANATOMICAL SITES}

Migle Gedgaudaite, Arturas Sukovas, Arnoldas Bartusevicius, Saulius Paskauskas, Daiva Vaitkiene, Ruta Jolanta Nadisauskiene, Adrius Gaurilcikas. Lithuanian University of Health Sciences; Obstetrics and Gynaecology

\subsection{6/ijgc-2020-ESG0.66}

Introduction/Background Lymph-node status is one of the prognostic factors related to the survival of patients with endometrial cancer (EC). However, systemic pelvic lymphonodectomy (PLN) is related to increased perioperative morbidity. A number of studies using different techniques have demonstrated the sentinel lymph-node biopsy (SLB) could be a better alternative to PLN in different patient groups. With evidence still lacking, SLB is considered an experimental method by major professional organisations like European Society of Gynaecologic Oncology. The aim of this study was to evaluate the adherence of the SLB procedure in a center with no previous experience of SLB in EC.

Methodology Prospective interventional study was performed in Lithuanian University of Health Sciences Hospital, Centre of Oncogynaecology in the period of 2018 March and 2020 July. 96 patients with histologically confirmed endometrioid endometrial carcinoma were included into the study. Indocyanine green (ICG) dye was used to map sentinel lymph-nodes using previously described technique. PLN was performed after SLB procedure for intermediate and high-risk patients.

Results Detection rate, timing and anatomical sites

The overall SL detection rate was $87.5 \%$ (bilateral in $63.5 \%$ (61/96), unilateral in additional $24.0 \%$ (23/96) of patients). The median time for the detection of the 1st SL was 35 minutes after injection of ICG (range 13-140 min), and 45 minutes (range 25-115 $\mathrm{min}$ ) for the 2nd (contralateral) one. The median number of SL removed was 2 (range $1-8)$. The most frequent sites for SLs were right external iliac area $(31.0 \%)$, left external iliac area $(24.2 \%)$, right internal iliac area (11.9\%) and left obturator fossa (11.3\%). $4.8 \%$ of SL mapped in paraaortic region.

SL metastasis rate Lymph node metastasis were found in 6 $(6.3 \%)$ patients and $4(4.4 \%)$ of them were detected by SLB. The sensitivity of SLB was $66.7 \%$ and negative predictive value $97.4 \%$. SLB has moderate - strong agreement with PLN (kappa coefficient 0.787, $\mathrm{p}<0.001$ ).

SL mapping failures SL mapping failed in $12.5 \%$ (12/96) of the patients. The factors that might be associated with mapping failure was age (73 vs. 64.5 vs. $62.8, \mathrm{p}=0.005)$ and present extragenital pathology $(100 \%$ vs. $60.9 \%$ vs. $57.4 \%$, $\mathrm{p}=0.019)$. 
Conclusion With the overall detection rate of $87.2 \%(63.5 \%$ bilateral and $24.0 \%$ - unilateral) we find SLB ICG procedure feasible. Most frequently SLs were detected in the external iliac region. Age and extragenital pathology are the statistically significant factors associated with the failure of SLB procedure.

\section{ROLE OF THREE-DIMENSIONAL TRANSVAGINAL ULTRASOUND AND DIFFUSION-WEIGHTED MAGNETIC RESONANCE IMAGING FOR ASSESSMENT OF MYOMETRIAL INVASION IN PATIENTS WITH LOW-RISK ENDOMETRIAL CANCER}

${ }^{1}$ Núria Carreras Diéguez, ${ }^{1}$ Isabel Matas, ${ }^{2}$ Crístian de Guirior, ${ }^{2}$ Meritxell Munman ${ }^{1}$ Pere Fusté, ${ }^{1}$ Núria Agustí, ${ }^{1}$ Ariel Glickman, ${ }^{1}$ Berta Diaz-Feijoo, ${ }^{1}$ Jaume Pahisa, ${ }^{1}$ Aureli Torne. ${ }^{1}$ Hospital Clínic de Barcelona; Gynecologic Oncology Unit; ${ }^{2}$ Hospital Clínic de Barcelona; Gynecology and Obstetrics

\subsection{6/ijgc-2020-ESG0.67}

Introduction/Background In patients with early-stage, grade 12, endometrioid endometrial cancer, preoperative assessment of myometrial invasion is essential to define the need of pelvic and paraaortic lymph node dissection. Our aim was to evaluate the role of three-dimensional transvaginal ultrasound (3D-TVUS) and diffusion-weighted magnetic resonance imaging (DW-RMI) for the assessment of myometrial invasion in patients with low-risk endometrial cancer.

Methodology We performed a single center retrospective study, including patients who underwent surgery for grade 1-2 endometrioid endometrial cancer, FIGO stage I-II, in Hospital Clínic de Barcelona between 2010 and 2019. We computed sensitivity, specificity, and predictive values of 3D-TVUS and DW-RMI, as well as of intraoperative frozen section pathological study of surgical specimen, for diagnosis of deep myometrial invasion $(\geq 50 \%)$. Definitive pathological analysis of surgical specimen was considered gold standard for diagnosis of deep myometrial invasion.

Results One hundred and fifty-three patients were included, $120(78.43 \%)$ patients presented myometrial invasion $<50 \%$ in postoperative analysis of surgical specimen and 33 (21.57\%) patients presented deep myometrial invasion. Sensitivity and specificity of 3D-TVUS for diagnosis of deep myometrial invasion was $68.8 \%$ and $80.5 \%$ respectively, while DW-RMI showed a sensitivity and specificity of $76.2 \%$ and $84.4 \%$. When combining both techniques (we considered that a patient had deep myometrial invasion when 3D-TVUS or DW-RMI - or both of them - showed deep myometrial invasion), sensitivity was $93.1 \%$ and specificity was $68.4 \%$. The proportion of patients with uterine fibroids was higher in the group of patients with false negative $(60 \%)$ or false positive $(39.13 \%)$ result in 3D-TVUS, although these results did not reach statistical significance. Regarding the intraoperative frozen section pathological study of surgical specimen, it showed a sensitivity of $75 \%$ with specificity of $96.4 \%$ for diagnosis of deep myometrial invasion.

Conclusion The combination of 3D-TVUS and DW-RMI offers a better sensitivity, higher than intraoperative frozen section pathological study of the surgical specimen, for the diagnosis of deep myometrial invasion in patients with early-stage, grade 1-2, endometrioid endometrial cancer. Such information may be useful in selecting patients who require lymph node dissection.

Disclosures The authors of this abstract have no disclosures.

\section{CLINICAL PARAMETERS PREDICTING RISK OF CONCURRENT INVASIVE CARCINOMA AND HIGH-RISK CARCINOMA IN PATIENTS WITH ENDOMETRIAL INTRAEPITHELIAL NEOPLASIA}

Nazlı Orhan', Raziye Melike Yildirim', Halise Meltem Batur', Utku Akgor², Nejat Ozgul', Murat Gultekin', Mehmet Coskun Salman'. 'Hacettepe University Faculty of Medicine; Department of Obstetrics and Gynaecology; ${ }^{2}$ Hacettepe University Faculty of Medicine; Department of Gynecological Oncology; Department of Obstetrics and Gynaecology

\subsection{6/ijgc-2020-ESGO.68}

Introduction/Background Endometrial intraepithelial neoplasia (EIN) is a premalignant lesion, but risk of concurrent endometrial adenocarcinoma (EAC) is also high. Although most patients with EIN diagnosed with concurrent EAC will have low risk disease, some will have high-risk disease who require. Clinical characteristics may help determine such patients.

Methodology Patients with a diagnosis of EIN who were operated at Hacettepe University Faculty of Medicine, Department of Obstetrics and Gynaecology were identified. The rate of concurrent EAC and high-risk EAC were determined. Preoperative characteristics were reviewed in order to determine the predictors of concurrent malignancy.

Results A total of 252 patients constituted study group. Mean age was 46.6 years and $43.7 \%$ were postmenopausal. $44.0 \%$ had co-existing one or more medical diseases while $14.3 \%$ had diabetes, $19.5 \%$ had hypertension, and $7.5 \%$ had both. The most common surgery was total hysterectomy with or without adnexal removal performed in $93.6 \%$ of patients. Frozen section was requested for $82.5 \%$ of patients. Final pathology revealed EAC in $17.5 \%$, but only $4.4 \%$ had highrisk disease. The accuracy of frozen section for predicting final pathology in terms of the presence or absence of EAC was $89.4 \%$. Patient with malignancy tend to be significantly older (47.4 vs. 54.1 years, $\mathrm{p}=0.02)$ and risk of malignancy was significantly higher in postmenopausal women $(9.2 \%$ vs $28.2 \%, \mathrm{p}<0.001)$ and in women with hypertension $(13.8 \%$ vs $32.7 \%, \mathrm{p}=0.02)$. similarly, patients with high-risk disease were significantly older (48.2 vs. 58.2 years, $\mathrm{p}=0.01$ ) and this risk was higher in postmenopausal women $(1.4 \%$ vs. $8.2 \%$, $\mathrm{p}=0.01)$ and women with hypertension $(3.0 \%$ vs. $10.2 \%$, $\mathrm{p}=0.04)$.

Conclusion Surgery is the mainstay of treatment in patients with EIN. During surgery, frozen section evaluation should be requested since a significant proportion of patients have concurrent EAC and frozen section is highly effective in determining these patients. Although rare, some patients may have concurrent high-risk endometrial carcinoma necessitating surgical staging. Both concurrent invasive carcinoma and high-risk disease are associated with older age, being in postmenopausal period, and having hypertension.

Disclosures No potential conflict of interest to declare.

\section{ENDOMETRIAL BIOPSIES: FOR WHOM AND WHEN?}

Bilal Esat Temiz, ${ }^{1}$ Esra Karataş, ${ }^{2}$ Utku Akgor, ${ }^{3}$ Murat Gultekin, ${ }^{3}$ Mehmet Coskun Salman, ${ }^{1}$ Nejat Ozgul. ${ }^{1}$ Hacettepe University Hospital; Obstetrics and Gynecology; ${ }^{2}$ Hacettepe University Faculty of Medicine; Department of Gynecological Oncology; Department of Obstetrics and Gynaecology; ${ }^{3}$ Hacettepe University Faculty of Medicine; Department of Obstetrics and Gynaecology

10.1136/ijgc-2020-ESGO.69 